DOI: https://doi.org/10.46296/rc.v3i6.0018

\title{
Factores que inciden en la trayectoria laboral de la mujer emprendedora
}

\section{Factors that affect the work path of women entrepreneurs}

\author{
Autora: Feijó-Cuenca Nilba Priscila \\ Universidad Técnica de Manabí (UTM). Portoviejo, Ecuador. \\ nilba.feijo@utm.edu.ec \\ Autor: Feijó-Cuenca Tito Eliecer \\ Universidad Técnica de Manabí (UTM). Portoviejo, Ecuador. \\ tito.feijo@utm.edu.ec \\ Autora: Quiroz-Peñarrieta Irlanda Isabel \\ Contador público autorizado. Portoviejo, Ecuador. \\ irisbel12@hotmail.es
}

\begin{abstract}
RESUMEN
Esta investigación tiene como propósito analizar el rol de la mujer emprendedora y los factores culturales que marcan su trayectoria laboral y el enfoque esencial de sacar adelante a su familia. A través de un trabajo analítico basado en estudios documentales e investigaciones desarrolladas por la Organización de las Naciones Unidas - Mujeres, se pudo conocer cómo las mujeres enfrentan el emprendimiento. En contraste con los criterios de expertos se pudo evidenciar que las características más comunes entre las entrevistadas están la gestión de tiempo, la asignación cultural de dar a la mujer la principal responsabilidad sobre las riendas del hogar y el cuidado de los hijos, la mayoría de los emprendimientos liderados por mujeres giran en torno a la gastronomía, la belleza y la comercialización de artículos diversos.
\end{abstract}

Palabras clave: emprendimiento, cultura, autoempleo, familia, género.

\begin{abstract}
This research aims to analyze the role of women entrepreneurs and the cultural factors that mark their career and the essential approach of moving forward with their family. Through an analytical work based on documentary studies and research developed by the United Nations Organization - Women, it was possible to know how women face entrepreneurship. In contrast to the criteria of experts, it was evident that the most common characteristics among the interviewees are time management, the cultural
\end{abstract}


assignment of giving women the main responsibility for the reins of the home and the care of their children, most of Women-led ventures revolve around gastronomy, beauty and the marketing of diverse items.

Keywords: entrepreneurship, culture, self-employment, family, gender.

\section{INTRODUCCIÓN}

Las principales aportaciones al desarrollo económico se generan a través de la actividad emprendedora, debido al sinnúmero de participaciones que surgen en los diferentes sectores por medio de la generación de negocios dinámicos, logrando que a nivel social haya una mayor cantidad de ingresos.

Parte fundamental de ese engranaje social es la familia, cuyos miembros constantemente tienen la responsabilidad de trabajar articuladamente para el bienestar de esta. En este entorno, la práctica de actividades responsables promueve el equilibrio y los lazos afectivos. El planeamiento de soluciones económicas que contribuyan a la satisfacción de las necesidades de la familia es una de las principales responsabilidades de los padres quienes tienen a su haber la jefatura del hogar.

En muchos hogares, la participación de los padres en la generación de ingresos para garantizar la sostenibilidad de las familias es continua. No obstante, se ha podido apreciar el surgimiento de nuevas estructuras familiares distintas a la tradicional, aquellas donde la madre es la principal fuente de ingreso a las familias.

Justamente, la presente investigación se enfoca en analizar la participación y presencia de la mujer en el entorno familiar y la forma en que ésta asume el desafío emprender cotidianamente para salir adelante, llevando consigo a quienes forman parte de ese núcleo.

\subsection{Oportunidades de Formación}

Un número importante de mujeres hoy en día son emprendedoras, algunas por tener una actividad que les permita independencia y solvencia económica y, en muchos casos, por la necesidad de sacar adelante a su familia. 
Otros aspectos importantes que destacan a la mujer en actividad empresarial, es la particularidad con que asume sus responsabilidades laborales y las combina con la administración del hogar, estudios y vida personal (Abello, 2012).

Si bien Heller (2010), afirma que tanto hombres como mujeres en tema de equidad de género tienen las mismas oportunidades para emprender, las mujeres presentan una actitud de preocupación constante por destacar en su ambiente laboral y preparación académica, con el fin de fortalecer su perfil académico y tener mayor oportunidad de insertarse en el mercado ocupacional con acierto.

En Ecuador es cada vez mayor el número de mujeres que se insertan a las universidades para obtener su título académico, observándose que las carreras centradas en las ciencias sociales y de la salud (administración, trabajo social, secretariado, docencia, enfermería, entre otras), son aquellas en las que su presencia es más marcada. Sin embargo, es muy importante observar cuales con las circunstancias en las que deben desenvolverse las mujeres a pesar de las muchas conquistas logradas a través de los años por los movimientos feministas.

\subsection{Emprendimiento y familia}

En el ámbito empresarial las mujeres incursionan con mucho entusiasmo, pero con poco capital, lo cual suele responder al temor natural femenino de la asunción de riesgo y también a las limitaciones que suelen tener como sujetos de crédito, por lo que la mayor asistencia económica generalmente proviene de fuentes no formales como los amigos, la familia, entre otros.

Según Chaganti (1986), además de la contribución que la familia ejerce como fuerza impulsora para la instauración de emprendimientos y su crecimiento, el sexo parece influir en la autopercepción de la capacidad de éxito y en el establecimiento de objetivos de crecimiento del negocio. En el caso de las mujeres el apoyo de la familia favorece el desempeño de los negocios.

En estudios realizados por Smith-Hunter y Leone (2010), en países latinoamericanos se pone de manifiesto la participación activa de las mujeres 
como generadoras de ingresos, en especial en hogares de menos recursos. Lo cual confirma que el emprendimiento tiene mayor incidencia en países de menor desarrollo económico en donde a su vez la actividad emprendedora de la mujer se visualiza especialmente en las clases populares, como factor de autoempleo y motivada por la necesidad.

Estos negocios en muchos casos son iniciativas de autoempleo, realizadas de forma independiente y con la colaboración de otros miembros de la familia, que sustituyen con frecuencia la posibilidad de una entrada formal al mercado laboral y les ofrecen mayor autonomía en los procesos de decisión familiar.

Para Delgado (2007) los individuos que adquieren energía emocional de este tipo de interacciones sociales están motivados positivamente hacia la actividad emprendedora y rechazan el rol del empleado, que va en contra de la creatividad en los negocios y la innovación. Goss (2005), a su vez menciona que el aprendizaje de la actividad empresarial en la familia puede ser un ejemplo, en una familia donde la actividad emprendedora se desarrolla satisfactoriamente porque sus miembros aprenden la naturaleza de los negocios e interaccionan con más confianza en sí mismos (Cárdenas, 2009).

\subsection{El equilibrio}

La importancia central que se le brinda a la familia está atribuida a la función de eje central y lugar de armonía desde donde se direcciona la sociedad con visión de hogar. La preocupación por alcanzar una medida entre familia y trabajo (Guerrero, 2013). El compromiso de la mujer hacia su familia la lleva a hacer frente a una multiplicidad de funciones para rendir con un ritmo de vida sumamente acelerado, volcado hacia el aprendizaje continuo, conocimiento del funcionamiento de los negocios desde su organización hasta su posicionamiento en un mercado. La capacidad para emprender, el conocimiento del negocio, la claridad de ideas y metas, la planificación y las decisiones, así como la creatividad e innovación, son aspectos que destacan en el género femenino, especialmente la capacidad de equilibrar los ámbitos de acción de la familia y del trabajo (Valencia, 2010). 


\section{METODOLOGÍA}

Desde la perspectiva de Bonilla y Rodríguez (1997), la investigación cualitativa es aplicable a situaciones sociales para explorarlas y tratar de hacer acercamientos generales sobre fenómenos específicos para llegar a su comprensión, por lo que se empleó un tipo de investigación cualitativa de carácter descriptivo, orientado a determinar los factores que giran en torno al emprendimiento femenino y el equilibrio que la mujer da en su vida cotidiana a la administración de los negocios y la familia.

Para la estructura del estado del arte se analizan trabajos realizados por los investigadores en relación con la mujer emprendedora y una investigación efectuada a hombres y mujeres en el Ecuador a través de ONU Mujeres, de esta manera se pretende lograr una aproximación hacia las acciones de las emprendedoras a partir de aspectos externos que influyen en su desempeño.

\section{RESULTADOS}

De acuerdo con el estudio realizado por ONU Mujeres a las mujeres y hombres del Ecuador (2012), tanto mujeres como hombres tienen iguales oportunidades de educación, destacando que, aunque con una mínima diferencia, los varones tienen un registro más alto en temas de formación académica.

Fig. 1. Población y hogares por nivel de instrucción.

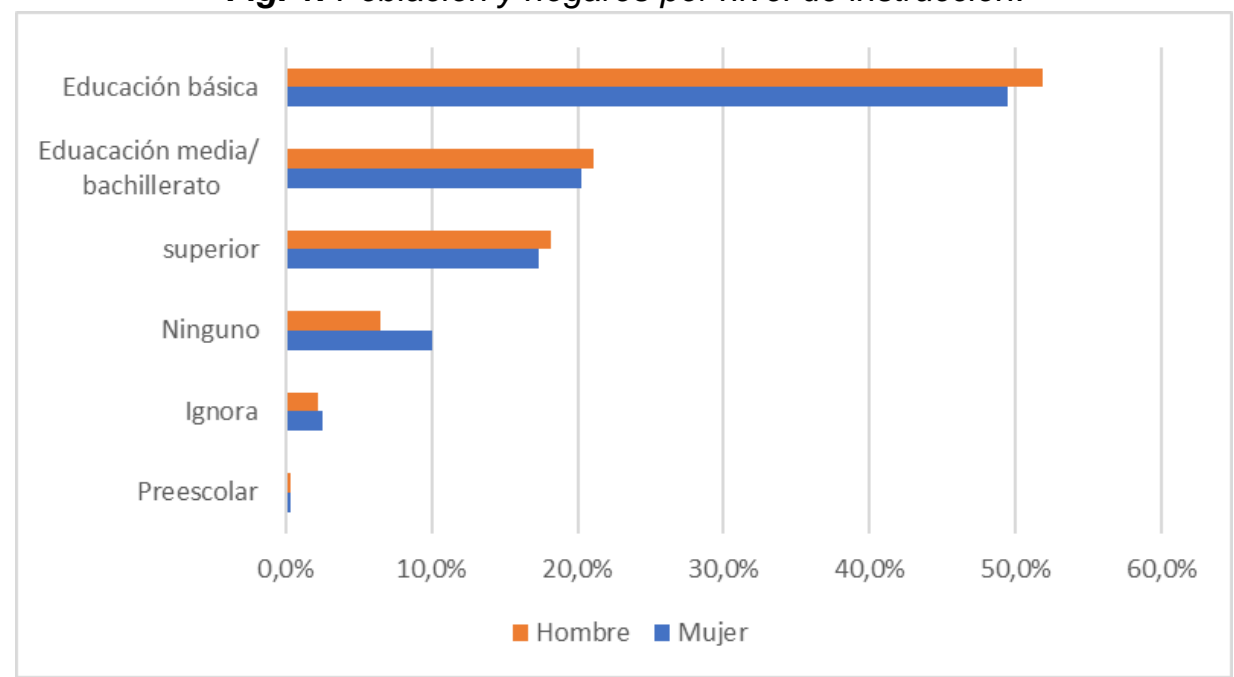

Fuente: INEC - Censo de Población y Vivienda 2010.

Elaborado por: Investigadores. 
Las razones por las cuales los ecuatorianos no han accedido a fuentes de educación formal son diversas, pero es importante señalar que en el caso de las mujeres, los embarazos a edades tempranas, los quehaceres del hogar y la falta de permiso de la familia, son los factores que tienen mayor incidencia, situaciones que evidentemente ponen en desventaja a este grupo de mujeres cuya falta de educación las destina a continuar realizando actividades invisibilizadas, poco valoradas y con baja o nula remuneración.

En el caso de los hombres, la dedicación al trabajo, el poco interés, la enfermedad y la discapacidad inciden en su falta de formación académica. Aspectos que inciden muy poco en la decisión de las mujeres.

Fig. 2. Razones de no asistencia a un establecimiento educativo.

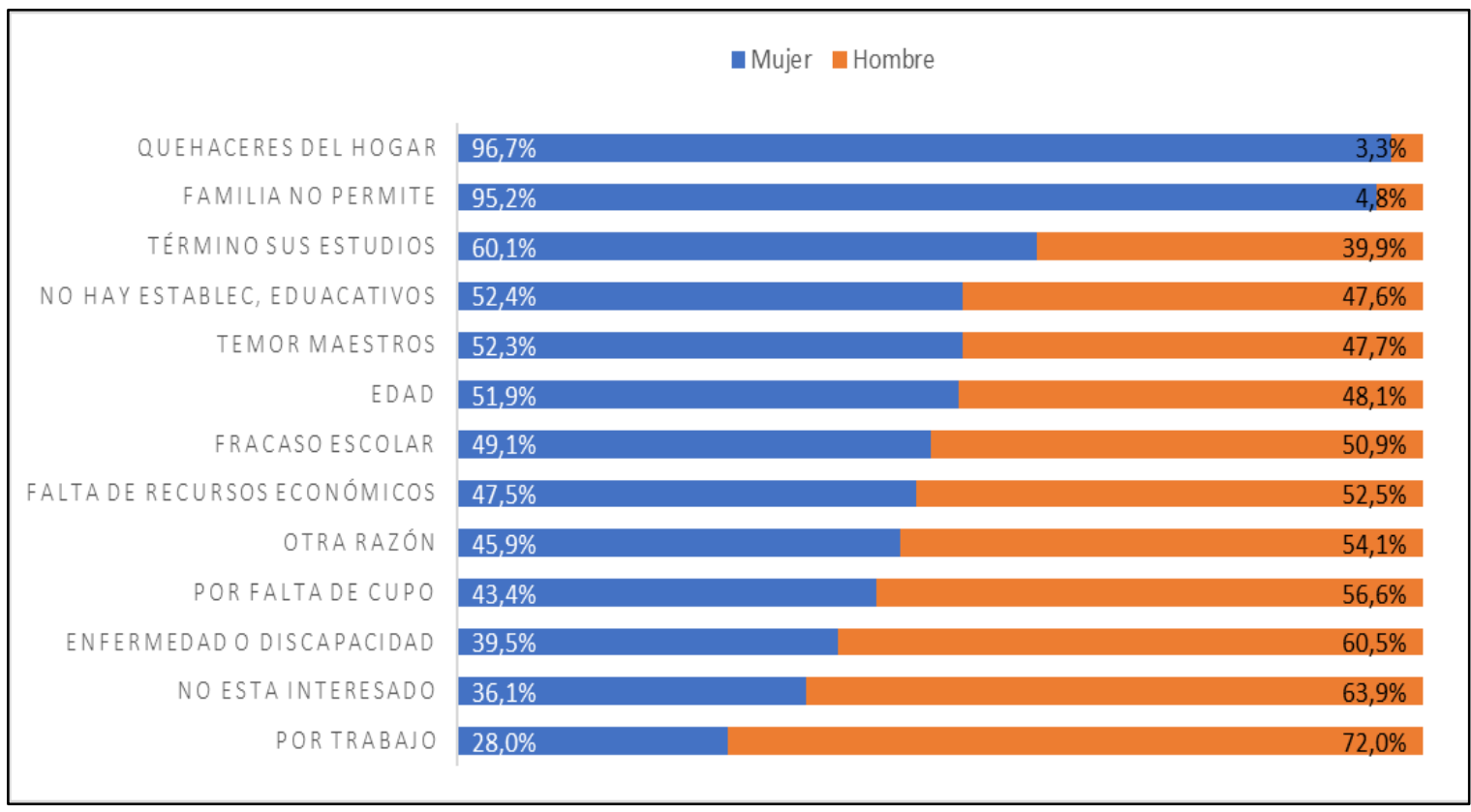

Fuente: INEC - Censo de Población y Vivienda 2010.

Elaborado por: Investigadores.

Las mayores actividades en las que se desempeñan las mujeres es en actividades de servicios domésticos, servicios sociales y de salud, hoteles y restaurantes, enseñanza. En contraste, a pesar de que muchas mujeres se preocupan por formarse académicamente, las oportunidades laborales las obligan a ocuparse principalmente en el servicio doméstico, con pocas opciones para poder abrirse un mejor camino. 
Fig. 3. Población ocupada, según rama de actividad.

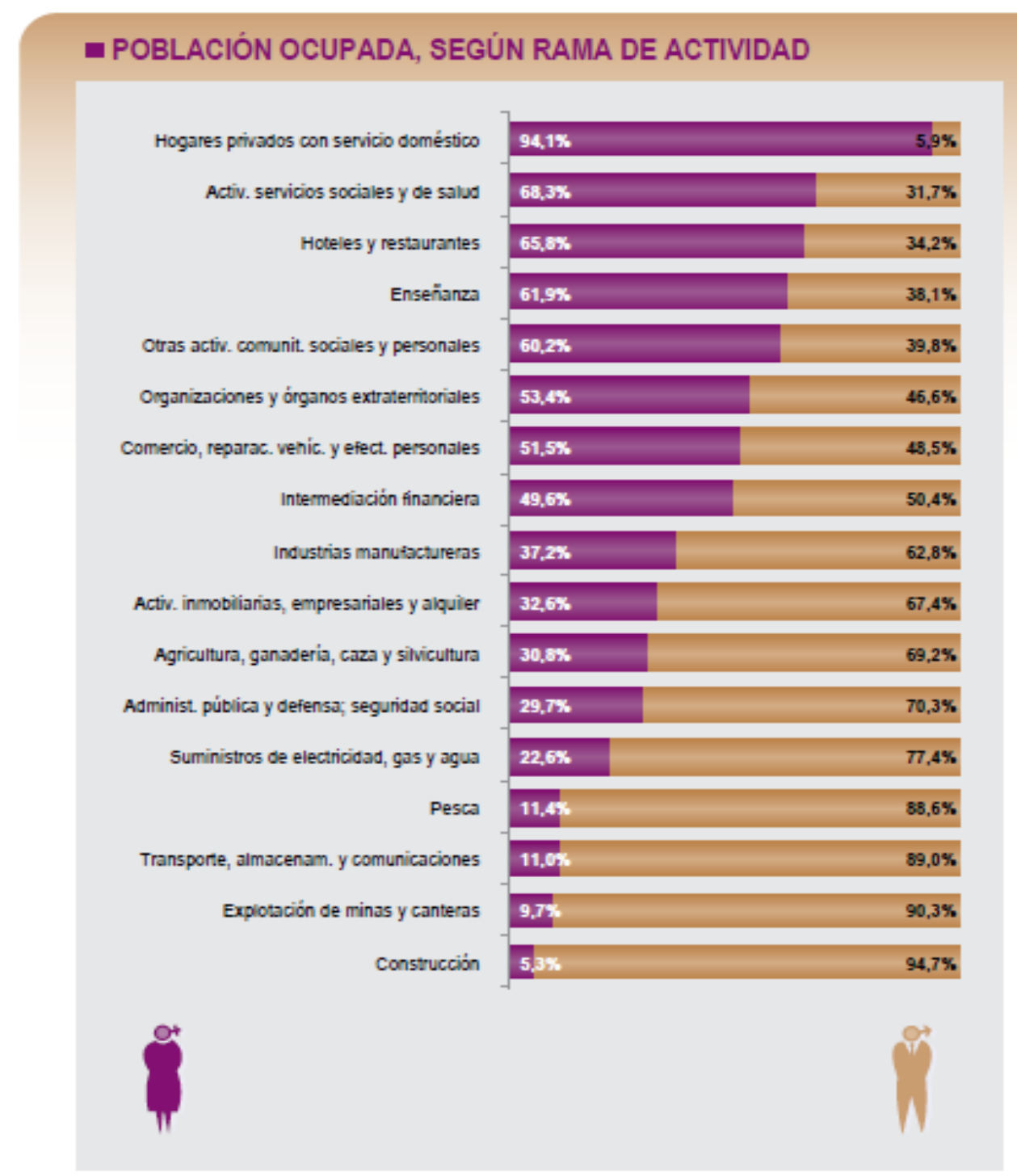

Fuente: INEC - Encuesta Nacional de Empleo, Desempleo y Subempleo -ENEMDU diciembre 2012 población de 10 años y más.

Elaborado por: ONU Mujer.

Esta parte de la estadística deja entrever que, aunque existen iguales posibilidades de educación para hombres y mujeres para acceder a centros educativos, existen limitaciones en algunas familias para que las niñas y jóvenes puedan estudiar, a su vez, el tema de la remuneración es muy marcado entre hombres y mujeres cuyo nivel de ingresos no es similar, fenómeno que se da de igual manera tanto en el sector urbano como en el sector rural. 
Tabla 1. Ingresos promedios de la población ocupada.

\begin{tabular}{|c|cr|rr|rr|rr|}
\hline \multirow{2}{*}{ Área } & \multicolumn{2}{|c|}{ Ingreso promedio en dólares } & \multirow{2}{*}{ Nacional } & \multirow{2}{*}{ Desigualdad } \\
\cline { 2 - 4 } & \multicolumn{2}{|c|}{ Mujeres } & \multicolumn{2}{|c|}{ Hombres } & & & \\
\hline Urbana & $\$$ & 421,00 & $\$$ & 524,00 & $\$$ & 483,00 & $\$$ & 80,30 \\
\hline Rural & $\$$ & 219,00 & $\$$ & 293,00 & $\$$ & 273,00 & $\$$ & 74,80 \\
\hline Nacional & $\$$ & 374,00 & $\$$ & 445,00 & $\$$ & 419,00 & $\$$ & 84,00 \\
\hline
\end{tabular}

Fuente: INEC - Encuesta Nacional de Empleo, Desempleo y Subempleo -ENEMDU diciembre 2012 población de 10 años y más.

Elaborado por: Investigadores.

La percepción que tiene la mujer de la familia la hace siempre pensar en el beneficio colectivo más allá del beneficio individual, por lo tanto, los ingresos que ésta genera sirven de ingreso directo hacia la educación y alimentación de la familia, cuando se trata de hogares unifamiliares.

En el caso de la estructura de las familias tradicionales, el aporte que la mujer da a la sostenibilidad del hogar es fundamental para la economía y sustentabilidad de los hijos, sin embargo, en el aspecto cultural puede generar conflictos porque aún se sigue perpetuando el hecho de que la responsabilidad por el sustento de la relación corresponde al hombre.

\section{CONCLUSIONES}

La mujer emprendedora tiene un gran valor para la sociedad y economía de un país, ya que forma parte de la Población Económica Activa, parte de su lucha constante está orientada a la busca del reconocimiento profesional y la decisión para sacar adelante, a través de sus esfuerzos a su familia, situación que la diferencia significativamente del sexo masculino y que hoy por hoy incide en el apoyo de organizaciones internacionales hacia el emprendimiento femenino.

En Ecuador los datos demuestran una importante incursión de la mujer en diferentes ámbitos (turismo, salud, educación, economía entre otros), lo que marca un sendero amplio de posibilidades para las futuras generaciones en donde hay mayor cantidad de escenarios para favorecer el desarrollo de 
habilidades y competencias empresariales para una acertada administración de sus negocios.

Las dinámicas vinculadas no solo al mejoramiento de capacidades sino de actitudes personales y fortalecimiento de valores, articulan una mejor fusión hacia el trabajo en equipo, liderazgo, habilidades gerenciales y comunicación, donde el proceso de formación sea sólo la primera etapa de una cadena de valor, en la que instituciones públicas y privadas puedan promover nuevas líneas de progreso para las mujeres.

\section{REFERENCIAS}

ABELLO, R. (2012). Infancia y conocimiento social. Barranquilla, Ediciones Uninorte.

AEH. (2013). Estudios de trayectoria escolar de la Maestría en Administración. Pachuca, Hidalgo: UAEH.

BONILLA, Elssy, RODRIGUEZ, Penélope. (1997). Más allá del dilema de los métodos. La Investigación en las ciencias sociales. Ediciones Uniandes. Edit. Norma. Buenos Aires.

CÁRDENAS, M. J., (2009) Mujeres emprendedoras y los beneficios del desarrollo sustentable en proyectos de inversión. Revista Ostara. Universidad Autónoma de Aguascalientes.

CHAGANTI, R. (1986). Management in Women-Owned Enterprises, Journal of Small Business Management.

DELGADO, M. Gómez, L., Romero A.M., \& Vázquez, E. (2007). Determinantes sociales y cognitivas en el espíritu emprendedor: un estudio exploratorio entre estudiantes argentinos. Universidad complutense de Madrid.

GOSS, David (2005). Emprendimiento y 'lo social': hacia una teoría de la deferencia-emoción.

https://journals.sagepub.com/doi/10.1177/0018726705055965

GUERRERO RAMOS, L. A., et. al. (2013). Construcción de un perfil de las mujeres emprendedoras en Torreón, Coahuila, México. Revista Internacional Administración \& Finanzas. 
HELLER, L. (2010). CEPAL. Recuperado el 12 de marzo de 2013, de CEPAL: http://www.eclac.org/publicaciones/xml/4/38314/Serie93.pdf

ONU, Mujer (2012). Mujeres y hombres del Ecuador en Cifras III.

SMITH-HUNTER, Andrea E.; LEONE, Joshua (2010). Evidence on the Characteristics of Women Entrepreneurs in Brazil: An Empirical Analysis.

VALENCIA SILVA, M. M. (2010). Perspectiva académica de la actividad emprendedora de las mujeres. En U. de Colima, Mujeres y empresa. Acercamiento multidisciplinario. Colima, México: Dirección General de Publicaciones de la Universidad de Colima. 